\title{
EL DEVÓNICO DE LA ZONA CENTROIBÉRICA
}

\author{
Miguel V. PARDO ALONSO' y Jenaro L.GARCÍA-ALCALDE \\ 'Departamento de Geología. Universitat de València; Dr. Moliner, 50. E-46100 Burjassot, València. e-mail: pardomv@uv.es
22 Departamento de Geología. Universidad de Oviedo; Jesús Arias de Velasco, s/n E-33005 Oviedo. Asturias. e-mail: jalcal-
de@asturias.geol.uniovi.es
}

Pardo Alonso, M.V. y García-Alcalde, J.L. 1996. El Devónico de la Zona Centroibérica [The Devonian of the Centroiberian Zone]. Revista Española de Paleontología, No Extraordinario, 72-81. ISSN 0213-6937.

\begin{abstract}
Within a synthesis study on Devonian stratigraphy, the boundaries of the Central-Iberian Zone (CIZ) are discussed. Devonian lithostratigraphic units are defined in the Almadén region and correlated along the Sierra Morena. Most parts of the Lower and Upper Devonian are paleontologically characterized. Nevertheless Lochkovian and Praguian are rather poorly represented and the Upper Famennian-Lower Carboniferous succession is very condensed. Middle Devonian rocks are lacking probably due to emergence during the Late Emsian. Correlation in the Sierra Morena region is largely based on a brachiopod-bivalve biozonation. As a special appendix the biostratigraphy and affinities of the Devonian of the northern Ossa-Morena Zone are discussed.
\end{abstract}

Keywords: Paleontology. Brachiopods. Stratigraphy. Devonian. Central-Iberian Zone. Northern Ossa-Morena Zone. Iberian Massif. Spain.

\section{RESUMEN}

Se discuten los límites de la Zona Centroibérica (ZCI) en el contexto de un estudio sintético sobre la estratigrafía del Devónico. En la región de Almadén se establece un esquema litoestratigráfico completo para el Devónico y sus unidades se correlacionan con otras del ámbito mariánico. Las asociaciones fósiles permiten identificar la mayor parte del Devónico Inferior y Superior; en cualquier caso, Lochkoviense y Praguiense están mal caracterizados y la sucesión Fameniense Superior-Carbonífero Inferior se muestra muy condensada. El Devónico Medio falta casi por completo, por la existencia de una extensa laguna estratigráfica, debida a emersión a fines del Devónico Inferior. La correlación en Sierra Morena durante el período Givetiense Superior-Frasniense Superior, se ha basado ampliamente en una biozonación de braquiópodos y bivalvos. Como apéndice separado, se discute la bioestratigrafía y afinidades del Devónico de la Zona Ossa-Morena septentrional.

Palabras clave: Paleontología. Braquiópodos. Estratigrafia. Devónico. Zona Centroibérica. Zona Ossa-Morena septentrional. Macizo Ibérico. España.

\section{LA ZONA CENTROIBÉRICA: LÍMITES}

Las antiguas Zonas "Galaico-Castellana" y "Lusooriental-Alcudiana”, en que Lotze (1945) dividió el Macizo Ibérico, fueron integradas por Julivert et al. (1974) en una sola unidad: la Zona Centroibérica (ZCI). Estas unidades, igual que las demás reconocidas en el Macizo Ibérico, se distinguen por sus características estratigráficas, metamórficas y plutónicas y sus límites coinciden generalmente con importantes accidentes tectónicos.

El límite meridional de la ZCI se sitúa, tradicionalmente, en el batolito de Los Pedroches, un extenso afloramiento de rocas graníticas, encajado en una estructura sinformal ocupada por depósitos carboníferos, que se prolonga en dirección ONO-ESE desde Castuera hasta Linares, donde desaparece bajo la cobertera del valle del Guadalquivir (Fig. 1). No obstante, desde el trabajo de Robardet (1976) muchos geólogos, principalmente estructurales, tienden a situarlo en la zona de cizalla Badajoz-Córdoba, una franja muy tectonizada al sur del batolito de Los Pedroches, aproximadamente paralela al mismo, desde Tomar (Portugal) hasta Córdoba.

Aunque la cizalla Badajoz-Córdoba, podría representar un límite paleogeográfico de mayor importancia que el bato- lito de Los Pedroches, en esta síntesis se ha optado por mantener la frontera original de la ZCI, debido a que las características bioestratigráficas y diastróficas de las regiones al $\mathrm{N}$ y $\mathrm{S}$ del sinclinal de Los Pedroches, son diferentes no sólo antes del Ordovícico sino también del Silúrico Superior al Carbonífero. El grado preciso de diferenciación bioestratigráfica no puede determinarse debido a que la documentación del "dominio" Obejo-Valsequillo-Puebla de la Reina (Delgado et al., 1977), situado entre los accidentes citados, es muy incompleta (generalmente sólo listados de fósiles) y se refiere, con frecuencia, a afloramientos aislados, de estratigrafía mal conocida.

Entre las diferencias estratigráficas principales entre el norte de Los Pedroches y el "dominio" Obejo-Valsequillo-Puebla de la Reina pueden mencionarse:

a) El Silúrico Superior al S de Los Pedroches, se desarrolla en facies de "calizas de Cardiola interrupta", calizas negras con nautiloideos y otros moluscos, singularmente el bivalvo Cardiola, ostrácodos y conodontos (vide, Hojas 1:50.000 de Valsequillo, Villaviciosa de Córdoba y Espiel); estas series son muy frecuentes en el resto de la Zona Ossa-Morena (Jaeger y Robardet, 1979), mientras al norte de Los Pedroches aparecen exclusivamente en su extremo noroc- 


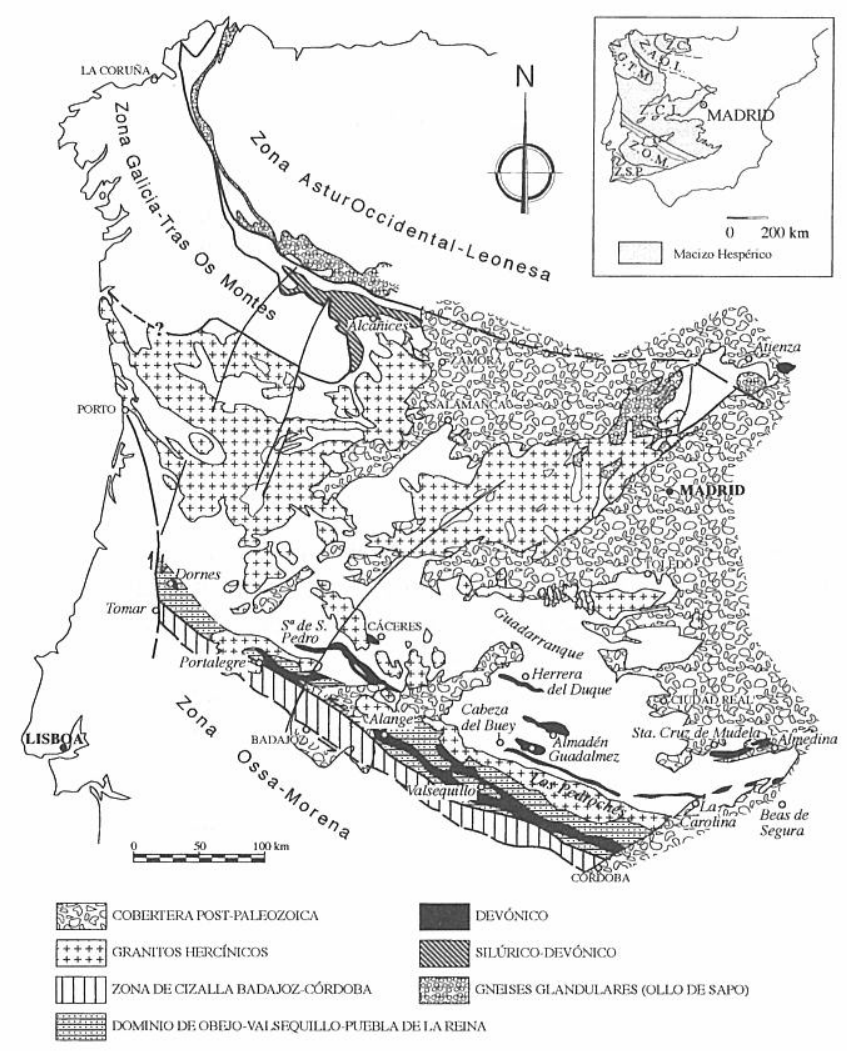

Figura 1. Mapa esquemático de la Zona Centroibérica. Afloramientos del Devónico en la zona y en áreas limítofres y localidades de referencia.

cidental (Sinclinal de Alcañices, Aldaya et al., 1976), muy alejados de Ossa-Morena.

b) El Devónico Inferior, suele estar bien representado en el "dominio" Obejo-Valsequillo-Puebla de la Reina del Lochkoviense al Emsiense, con asociaciones fósiles características de braquiópodos, corales y trilobites, mientras al $\mathrm{N}$ de Los Pedroches, el Lochkoviense y el Praguiense están mal caracterizados paleontológicamente.

c) La amplia laguna estratigráfica reconocida hace tiempo en el Macizo Ibérico meridional (Puschmann, 1967; Pardo Alonso y García-Alcalde 1984a, 1984b), parece de extensión diferente en las regiones comparadas, abarcando al norte, desde la parte más alta del Emsiense al Givetiense terminal o al Frasniense Inferior, mientras al sur puede alcanzar incluso el Fameniense Superior en parte del "dominio" Obejo-Valsequillo-Puebla de la Reina. En efecto, en este área no se conocen las abundantes y características asociaciones fósiles de espiriféridos, rinconélidos y estrofoménidos frasnienses, que al norte de Los Pedroches se extienden, al menos, desde la Sierra de San Pedro hasta Sierra Morena oriental (Fig. 1). La extensión máxima de la laguna en esta región sería, así, similar a la observada en el sinclinal de Valle, en la provincia de Sevilla, en plena Zona Ossa-Morena (Racheboeuf y Robardet, 1986; Weyant et al., 1988).

d) El Fameniense, característicamente desarrollado al norte en pizarras nodulosas y calizas pelágicas, con abundantes ammonoideos (Fms. Guadalmez y Casa de la Vega, ver luego), sólo se conoce hasta ahora en el sur, con cierta seguridad, en facies de areniscas bioclásticas, con abundantes braquiópodos.

Podría ser que las diferencias señaladas tuviesen meramente un significado batimétrico de escala regional limitada, pero eso habría que probarlo. En cualquier caso, aunque de momento se opte por mantener el límite meridional de la Zona Centroibérica en su posición original, en esta síntesis se incluye un apéndice con los datos bioestratigráficos conocidos inmediatamente al sur de Los Pedroches.

La parte noroccidental del límite septentrional de la ZCI lo marca tradicionalmente el llamado antiforme del "Ollo de Sapo" (pero ver Martínez Catalán et al., 1990, y Díez Balda et al., 1990), con núcleo formado por porfiroides, interpretados como precámbricos (Julivert, et al. 1974). La llamada "serie de San Vitero" o Fm. San Vitero (Aldaya et al., 1976; Gutiérrez-Marco et al., 1990), que aflora en el sinclinal de Alcañices-Carbajales (prov. de Zamora) (Fig. 1), se encuentra al sur de dicho límite y ha proporcionado fósiles devónicos del Lochkoviense al Emsiense Superior. Las facies y el carácter de las asociaciones fósiles: conodontos y dacrioconáridos hercínicos (Carls, en Aldaya et al., 1976; Truyóls-Massoni y Quiroga, 1981), son más próximos a los de la Zona Asturoccidental-Leonesa (Guadarrama oriental, Cadenas Ibéricas) y Dominio Palentino, que a los de la ZCI meridional, lo que indicaría condiciones transicionales entre ambas Zonas durante el Devónico.

La prolongación del límite septentrional de la ZCI está oculta, en buena parte, bajo las rocas cenozoicas de la cuenca del Duero y nunca se definió, con claridad, en la sierra de Guadarrama. Esta imprecisión es, sin duda, la causa de que el Guadarrama oriental se haya situado tanto en la Zona Asturoccidental-Leonesa (Lotze, 1945; Julivert et al., 1974; Julivert et al., 1983; Carls, 1988, inter al.) como en la ZCI (Gutiérrez-Marco et al., 1990; Díez-Balda et al., 1990; Robardet et al., 1993). Esta región, extendiéndose aproximadamente entre las localidades de Atienza, Cercadillo y Riba de Santiuste (Fig. 1), presenta una sucesión paleozoica muy potente. La secuencia es muy fosilífera y aparentemente continua, al menos desde el Ludloviense al Emsiense (Bultynck y Soers, 1971). Los fósiles devónicos ilustran, sin ambiguiedad, la presencia del Lochkoviense, Praguiense y Emsiense, si bien la parte alta de este último Piso no está representada por causa de la erosión pre-triásica. Las facies y las asociaciones fósiles son estrechamente comparables a las del Dominio Palentino y Cordillera Ibérica Oriental, con correlaciones de la máxima precisión entre las tres regiones (Carls, 1988; García-Alcalde et al., 1990), y difieren radicalmente de las de la ZCI. En consecuencia, el área discutida pertenecería a la ZAOL y su estudio excede los límites del presente trabajo.

\section{BREVE RESEÑA HISTÓRICA}

La ZCI, sobre todo su parte meridional, ha sido objeto de numerosas investigaciones debido a la minería. Sin embargo, la complicación tectónica y los malos afloramientos han obstaculizado bastante el conocimiento del área.

El trabajo bioestratigráfico más importante del siglo pasado, fue el de Prado, Verneuil y Barrande (1855), donde se citan, describen y figuran numerosas especies del Paleozoico, en particular del Devónico. 
De la primera mitad de este siglo, son destacables las observaciones de Groth $(1911,1914)$, sobre el Devónico de Guadalmez, y Müller (1929), quién describió las asociaciones frasnienses de Almadén y Guadalmez y sugirió un esquema biozonal en el que se inspiraría la biozonación propuesta posteriormente por los autores (Pardo Alonso y García-Alcalde, 1984b).

Tras una prolongada interrupción, los geólogos alemanes reiniciaron las investigaciones en la ZCI. Entre éstas, destacan las de Puschmann (1967, 1970), que puso de relieve la existencia de una importante laguna estratigráfica, comprendiendo el Devónico Medio y describió con detalle la estratigrafía del sinclinal de Herrera del Duque (1970), aportando muchos datos paleontológicos (en especial del Devónico Inferior), entre los que se incluyen las primeras citas de conodontos. La escuela de Münster realizó una gran cantidad de tesis y tesinas, la mayoría inéditas, que se resumen en Walter (1977) (Ver también Maass, 1961; Butenweg, 1968; Kettel, 1968).

Los franceses de la Universidad de Orsay consagraron también numerosos trabajos a la región, pero en su mayor parte permanecen impublicados.

Hacia la misma época apareció la obra de Almela et al. (1962) sobre la geología de la concesión minera de Minas de Almadén, la primera panorámica general del Paleozoico y punto de partida de los estudios modernos del área.

Julivert et al. (1983), realizaron una interesante síntesis del Devónico del Macizo Ibérico. Esta obra, precedió a las de los autores (Pardo Alonso y García-Alcalde, 1984a, 1984b; García-Alcalde et al., 1984), donde se analiza con detalle la estratigrafía del Devónico de los sinclinales más meridionales y su contenido fosilífero, y se propone una biozonación del Frasniense, extensible prácticamente a todo el dominio centroibérico meridional.

Por último, Gutiérrez Marco et al. (1990) presentaron una nueva síntesis del Paleozoico centroibérico, que incluye una visión amplia sobre el Devónico. Contiene una revisión reciente y sistematizada de la bibliografía sobre el Devónico centroibérico, y a ella remitimos a quien estuviera interesado en una reseña bibliográfica más detallada.

En el presente trabajo se incorporan los datos conocidos sobre las series y fósiles devónicos de la ZCI y otros nuevos pertenecientes a la tesis doctoral en curso de uno de nosotros (M.V.P.A.) y a informes del Proyecto MAGNA 1:50.000 (J. L. G. -A. et al.).

\section{ESTRATIGRAFÍA DEL DEVÓNICO}

Las sucesiones devónicas más completas de la ZCI se localizan en los sinclinales de Herrera del Duque, Almadén-Garlitos, Guadalmez y borde septentrional del de Los Pedroches (Fig. 1). Hasta ahora en este área sólo existían cuadros estratigráficos locales (Puschmann, 1970, en Herrera del Duque; Almela et al., 1962 y Pardo Alonso y García-Alcalde, 1984a y b, en los sinclinales meridionales). Uno de nosotros (M. V .P. A.), ha realizado recientemente un análisis integral de la región que le ha permitido establecer una litoestratigrafía generalizada (Figs. 2, 3), cuyas unidades se definirán formalmente más adelante. La escala litoestratigráfica conserva, con matizaciones, algunas de las unidades de Puschmann (1970) y propone otras nuevas.

Las unidades del Grupo Cerro Escudero (Fig. 3) equivalen, en parte, a las Formaciones "Guadarranque", "Cabezuelas" y "Cerro Herrumbre", definidas en el sinclinal de Guadarranque (Fig. 1) por Rodríguez Núñez et al. (1989). La Fm. Cerro Herrumbre no es bien conocida, pero la descripción de los autores citados, coincide en gran parte con las características litológicas de la Fm. Doradillo.

La conocida "Cuarcita de base" de Almela et al. (1962), equivale a la "Cuarcita Risquillo" de Puschmann (1970), nombre que se adopta aquí.
Herrera del Duque

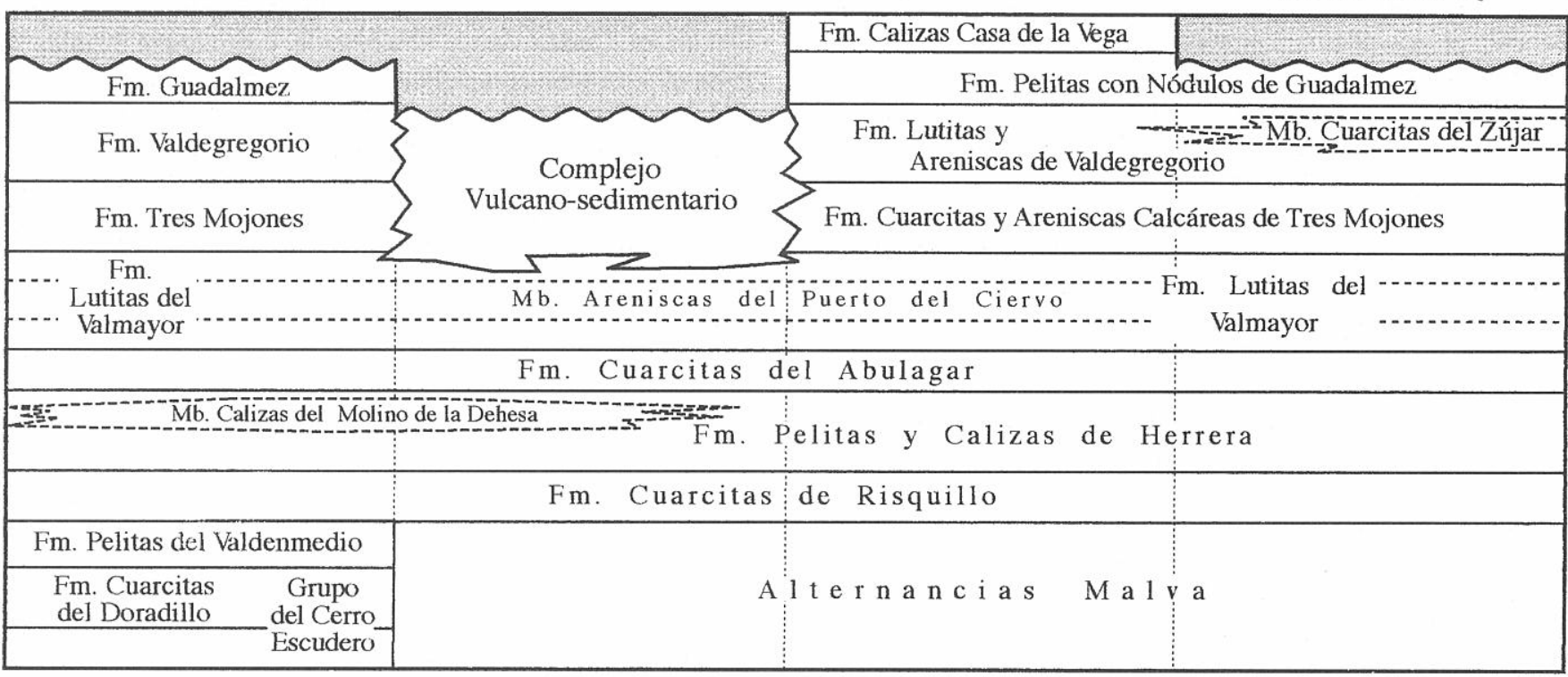

Figura 2. Distribución de las unidades litoestratigráficas establecidas en la Zona Centroibérica meridional [Fm. = Formación Mb. = Miembro]. 




Figura 3. Columna sintética del Devónico centroibérico meridional. Rango de los taxones más representativos. 
El Miembro Molino de la Dehesa de la Fm. Herrera (Fig. 3) presenta, en Herrera del Duque, calizas con estromatolitos, las primeras de este tipo que se han descrito en el Devónico centroibérico (Pardo Alonso y De Renzi, 1993).

El rasgo estratigráfico más característico del Devónico centroibérico es la existencia de una laguna estratigráfica que comprendería, como mínimo, prácticamente todo el Devónico Medio. Puschmann (1967) la interpretó como una detención en la sedimentación. Oczlon (1990), por su parte, invocó la existencia en la zona de corrientes de contorno para explicar el hiato sedimentario. Sin embargo, las condiciones litológicas sugieren, más bien, una emersión a fines del Devónico Inferior. Así, el techo de la Fm. Herrera en el sinclinal de Guadalmez, está formado por lutitas y algunas areniscas, con laminación lenticular y flaser, fuertemente bioturbadas (burrows verticales), con frecuentes restos vegetales flotados a techo de ripples linguoides. En el mismo sinclinal, la base de la Fm. Abulagar incluye niveles de conglomerados y areniscas de grano grueso con estratificación cruzada y bases erosivas (Fig. 3). Estas rocas parecen derivar de sedimentos propios de medios de transición, como las llanuras de marea, o incluso de canales fluviales, lo que hace muy dudosas las supuestas corrientes de contorno, típicas de medios marinos profundos. Hacia el SW (Ossa-Morena nororiental) la laguna estratigráfica está ligada a una deformación intradevónica con pliegues asociados (Herranz Araújo, 1994) que conlleva el depósito en discordancia del Devónico Superior sobre un sustrato erosionado; en principio, la deformación no es tan evidente en nuestra zona, aunque no se descarta que la emersión mesodevónica esté en parte asociada a ella.

El Devónico de la ZCI es, salvo excepciones, poco fosilífero. Este hecho es patente en el Devónico Inferior, sobre todo en los sinclinales más meridionales, y menos notorio en el Devónico Superior, donde las cuarcitas y las lutitas son, a veces, lumaquélicas.

Los fósiles más frecuentes son de invertebrados marinos, principalmente braquiópodos y bivalvos, con claro predominio de los primeros. Aparecen también crinoideos (generalmente muy desarticulados), briozoos, trilobites, ostrácodos, corales, tentaculitoideos, cefalópodos, gasterópodos, serpúlidos y conodontos. Los restos vegetales son raros, y se encuentran, sobre todo, en el entorno de la gran laguna estratigráfica mesodevónica y en algunos nódulos de la Fm. Guadalmez; en los estromatolitos se han encontrado también filamentos de algas (Pardo Alonso y De Renzi, 1993).

Salvo en ciertos niveles de calizas arenosas, los fósiles aparecen como moldes, internos y externos, debido principalmente a la disolución de las estructuras esqueléticas por aguas de meteorización. En cuarcitas procedentes de testigos de sondeos, se observa que las conchas carbonatadas se conservan bien en profundidad. Es posible, sin embargo, que en las pelitas la disolución se haya producido durante la diagénesis.

Las facies tafonómicas son variadas y no es posible generalizar. En los yacimientos más conocidos del Frasniense, predominan facies de conchas desarticuladas (valvas aisladas, artejos sueltos, pigidios y cefalones separados, briozoos fragmentados), de tamaño homogéneo, muchas veces con signos de reelaboración (rellenos de material lutítico en las concavidades), en ocasiones muy fragmentadas; no es raro encontrar conchas orientadas por corrientes (partes convexas hacia arriba y ápices o umbos apuntando en la misma dirección), o apiladas y encajadas unas con otras. Las litofacies consisten en areniscas y cuarcitas, de grano fino, con bases generalmente erosivas, huellas de impacto y arrastre a muro (prod, bounce), una cierta granoselección positiva y a veces estructuras hummock en el techo. Estas capas se intercalan entre limolitas y pelitas bioturbadas y, en conjunto, pueden interpretarse como tempestitas, que concentran conchas acumuladas en niveles más antiguos, erosionados durante la tormenta; todo ello en el marco de una plataforma siliciclástica, situada entre el nivel de base del oleaje normal y el de tormenta. La mayoría de los niveles calcáreos con fósiles del Devónico Inferior tendrían un origen similar, pero en el ámbito de una plataforma carbonatada.

Algunos niveles lutíticos presentan fósiles excepcionalmente bien conservados, de tamaños variados, con frecuencia articulados (especialmente braquiópodos). Se trataría, en este caso, de entidades acumuladas en el propio lugar de producción y los componentes de la asociación formarían parte, con toda probabilidad, de la misma comunidad original.

La mayoría de entidades conservadas corresponden a restos de animales bentónicos. Salvo en casos muy concretos (calizas del Emsiense, Fig. 3), el número de especies por capa es muy bajo aunque el de individuos pueda ser elevado. Sin evaluar el sesgo tafonómico, las características apuntadas indicarían condiciones ambientales algo restringidas, con frecuentes aportes detríticos removilizados por las tormentas.

El límite Silúrico-Devónico es de localización imprecisa. Los primeros fósiles devónicos aparecen en el tercio inferior de la Fm. Doradillo (Fig. 3). Se trata de trilobites identificados como Trimerus cf. acuminatus (com. pers. M. Arbizu), del Lochkoviense. En los mismos niveles hay también pequeños braquiópodos rinconélidos, cuyo estado de conservación no permite una determinación precisa, pero que recuerdan a formas de la transición Silúrico-Devónico. Pardo Alonso y García-Alcalde (1984b) asignaron también al Lochkoviense Microsphaeridiorhynchus ? sp. de la base de la Fm. Risquillo. Por ello, el límite entre ambos Sistemas podría situarse en un nivel indeterminado del Grupo Cerro Escudero (Fig. 3). La importancia de estos datos es muy grande. En efecto, la falta de información bioestratigráfica había hecho sospechar a algunos autores la existencia de una laguna entre el Silúrico y el Devónico, comparable a la del Devónico Medio (Carls, 1988), con las implicaciones paleogeográficas correspondientes.

El techo de la Fm. Risquillo es algo más fosilífero, con braquiópodos del Praguiense y otros de afinidades emsienses (Fig. 3). Calvo (1993), basándose en conodontos, señala que el Praguiense alcanzaría, al menos en Herrera del Duque, las primeras intercalaciones carbonatadas de la Fm. Herrera, unos metros sobre la Fm. Risquillo (Fig. 3).

El Emsiense es el Piso mejor caracterizado del Devónico Inferior de la ZCI. La Fm. Herrera presenta abundantes fósiles de dicha edad, con braquiópodos, corales, tentaculitoideos (Tentaculites spp. y Viriatella aff. procera) y conodontos (Fig. 3). La transición Emsiense Inf./Sup., se produciría en el Miembro Molino de la Dehesa. La parte alta de la Fm. Herrera pertenecería al Emsiense Superior terminal, de acuerdo con la presencia de Arduspirifer gr. mosellanus dahmeri.

La mayor parte del Devónico Medio no puede caracterizarse paleontológicamente, posiblemente a causa de la gran 
laguna estratigráfica a la que se ha aludido anteriormente. De todos modos, entre las últimas asociaciones de fósiles emsienses y las siguientes suele haber algunos metros de serie del techo de la Fm. Herrera y de la base de la Fm. Abulagar, de edad desconocida, que proporcionaron sólo restos vegetales indeterminables.

La definición formal de las unidades cronoestratigráficas del Devónico, quizás entrañe el que asociaciones fósiles tradicionalmente consideradas en la ZCI como frasnienses hayan de reinterpretarse como del Givetiense Superior. En esta situación se encontrarían, por ejemplo, las de la Fm. Abulagar (Fig. 3). En el área de Alange, fuera de la ZCI en el sentido que aquí se le da, hay indicios aún más claros de la presencia del Givetiense Superior, como luego se indicará.

El posible Givetiense Superior y el Frasniense están generalmente bien caracterizados por abundantes fósiles, de manera que ha sido posible establecer una biozonación pluritaxónica (bivalvos y braquiópodos) (Pardo Alonso y García-Alcalde, 1984b), muy útil para la correlación en todo el ámbito meridional de la ZCI. Las biozonas definidas son las siguientes:

a) Cenozona Bivalvos. Caracterizada por numerosas especies de bivalvos, generalmente de gran tamaño: Lyriopecten gilsoni y otros pterinopectínidos, Cypricardella oblonga, Leptodesma almadenensis y Ctenodonta robustella, entre otros. La asociación se desarrolla típicamente en la parte media-baja de la Fm. Abulagar (Fig. 3), y se ha reconocido en los sinclinales de Almadén, Guadalmez y flanco norte de Los Pedroches (Fig. 1). Considerada inicialmente como de edad Frasniense Inferior podría corresponder, en términos actuales, al Givetiense Superior.

b) Biozona de extensión Cyphoterorhynchus. Caracterizada por la presencia de los braquiópodos Cyphoterorhynchus domenechae y C. marianus (Fig. 3). En contra de lo que se había supuesto en su momento (Pardo Alonso y García-Alcalde, 1984b, p. 92), los datos más recientes muestran que la aparición de la primera de las especies se produce antes que la de la segunda. La biozona se desarrolla en la mitad superior de la Fm. Abulagar (Fig. 3), y ha sido identificada en Herrera del Duque, Almadén, Guadalmez, flanco norte de Los Pedroches (excepto en su extremo oriental) y, con dudas, en la parte española del área de La Codosera-Portalegre (Fig. 1). La edad correspondería, tal vez, al Givetiense Superior o a la Cronozona de conodontos Asymmetricus Inferior (=Transitans, en términos actuales).

c) Biozona de extensión coincidente Alvarezi-Jordani. Caracterizada por la presencia conjunta de Douvillinoides? alvarezi y Eoschuchertella jordani. Se desarrolla típicamente en la base del Mb. Puerto del Ciervo (Fig. 3) en el sinclinal de Almadén (Fig. 1). Su edad correspondería quizás a parte de las Cronozonas Asymmetricus inferior y media (=Transitans y Punctata, en términos actuales).

d) Biozona de extensión Almadenensis. Se solapa en parte con la anterior, y está caracterizada por la presencia de Apousiella almadenensis. Junto a la especie zonal, suelen aparecer Productella subaculeata (sobre todo en la parte media de la biozona) y Douvillina delta y D. radiata (hacia la parte alta). Se desarrolla típicamente en el Mb. Puerto del Ciervo (Fig. 3) y presenta la distribución más amplia de todas las definidas en la ZCI, habiendo sido reconocida en Herrera del Duque, Almadén-Garlitos, Guadalmez, Los Pedroches,
Sierra de San Pedro y La Codosera-Portalegre, y posiblemente también en Sierra Morena oriental (Fig. 1). La biozona pertenecería, en parte, a la Cronozona Asymmetricus media (= Punctata, en términos actuales).

e) Biozona de extensión Muelleri. Definida por la presencia de Pradochonetes muelleri. La especie zonal, abundante en los niveles lutíticos, está acompañada típicamente por Apousiella bouchardi, Ripidiorhynchus cf. kotalensis y Cyrtospirifer almadenensis. En las facies areniscosas la especie zonal es rara, pero los elementos acompañantes se hacen más abundantes y permiten aún buenas correlaciones. La biozona se distribuye típicamente en las Fms. Valmayor, Tres Mojones y parte basal de Valdegregorio (Fig. 3). La edad correspondería, quizás, a parte de las Cronozonas Asymmetricus media $y$ superior (= Punctata y parte baja de Hassi Inferior, en términos actuales).

Por encima de la última biozona los fósiles se hacen raros. En el Mb. Zújar de la Fm. Valdegregorio (Fig. 3), se encontraron restos muy fragmentados de ammonoideos (Tornoceras? sp.) y Buchiola kaisini, en areniscas bioclásticas, del Frasniense Superior o del Fameniense Inferior. Sin embargo, algo más arriba, han aparecido tentaculitoideos (Nowakia sp.) y braquiópodos (Spinulicosta? sp.) como mucho del Frasniense.

De acuerdo con García-Alcalde et al. (1984) y Oliveira et al. (1986), el paso Frasniense-Fameniense se produciría en la base de la Fm. Guadalmez, donde hay una característica asociación fósil de ammonoideos y bivalvos (Fig. 3). La Fm. Guadalmez coincide en edad, facies y contenido fosilífero con otras unidades de la Península Ibérica (Fm. Fueyo, de la Zona Cantábrica; Mb. superior de la Fm. Hoya y Fm. Fuenpudrida, de la Cordillera Ibérica), Francia (parte inferior de las "Pizarras de Porsguen", en Bretaña occidental) y Alemania ("Capas de Nehden", en el Eifel), que representan una importante transición litológica y faunística indicando, tal vez, el Evento Kellwasser.

El Fameniense se encuentra aparentemente muy condensado en el tercio inferior de la Fm. Casa de la Vega, donde se suceden en pocos metros las Biozonas de ammonoideos Cheiloceras (doII) y Wocklumeria (doVI) (Fig. 3). Lo mismo sucede con el Carbonífero Inferior, representado en la misma formación por conodontos del Turnesiense Superior (Fig. 3).

El Devónico aflora al NO y al SE de la región de referencia, en núcleos sinclinales, con sucesiones comparables, aunque menos conocidas, a las que se han descrito (Figs. 1, 4).

En el área situada entre Santa Cruz de Mudela, Almedina y el río Guadalmena, al N de Beas de Segura, en Sierra Morena oriental (Fig. 1), el Devónico es muy areniscoso y poco fosilífero. La sucesión, denominada "Estratos de San Pablo" por Butenweg (1968), consta de dos potentes unidades arenoso-cuarcíticas (de más de $350 \mathrm{~m}$ de espesor cada una), separadas por un intervalo lutítico de algo menos de $100 \mathrm{~m}$, con varios niveles conglomeráticos en la parte inferior y media (Figs. 1, 4). La unidad inferior podría correlacionarse en parte con la Fm. Risquillo. En el techo de la misma, Kettel (1968) identificó fósiles, probablemente de Edad Praguiense. Sin embargo, el gran espesor y la proximidad estratigráfica a las pizarras silúricas, hace probable que comprenda también niveles equivalentes a las Fms. Valdenmedio y Doradillo. Gutiérrez-Marco et al. (1990) han sugerido que los niveles de conglomerados podrían corresponderse 


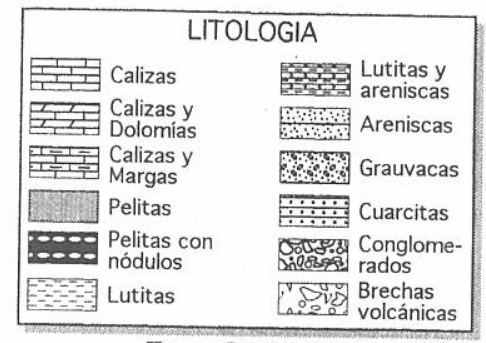

Zona Ossa Morena

(Dominio Obejo-Valsequillo)

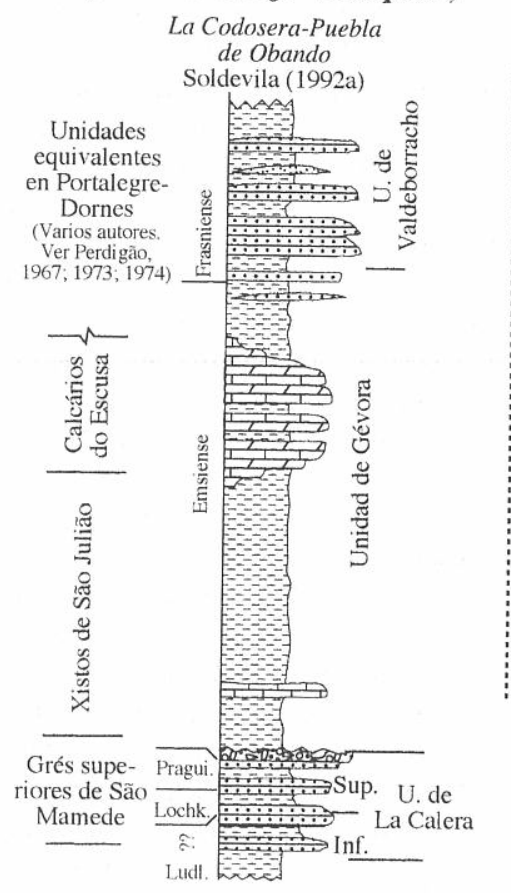

Extremadura Centro-Occidental/Beira Baixa
Zona Centroibérica Meridional



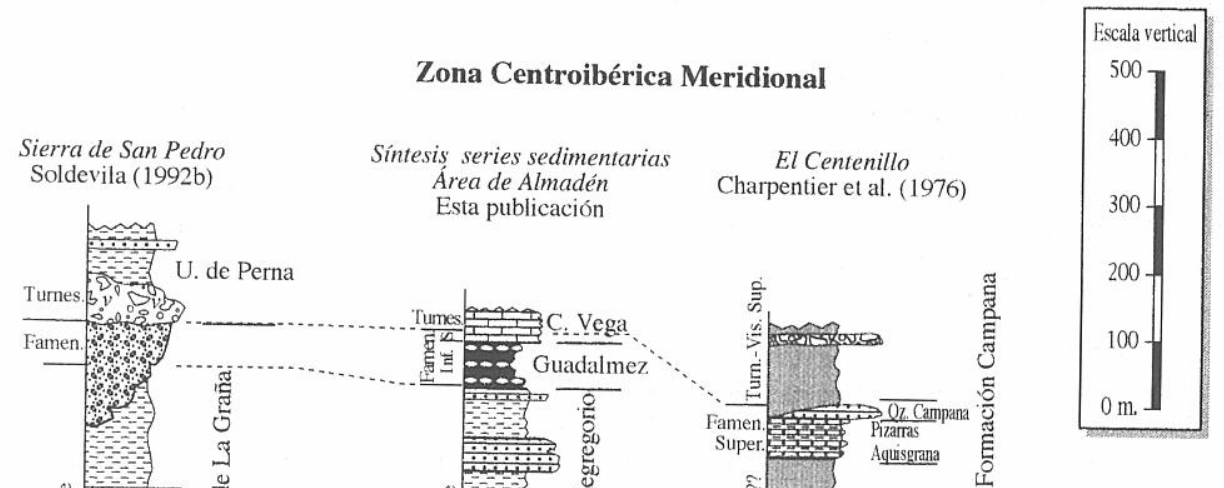

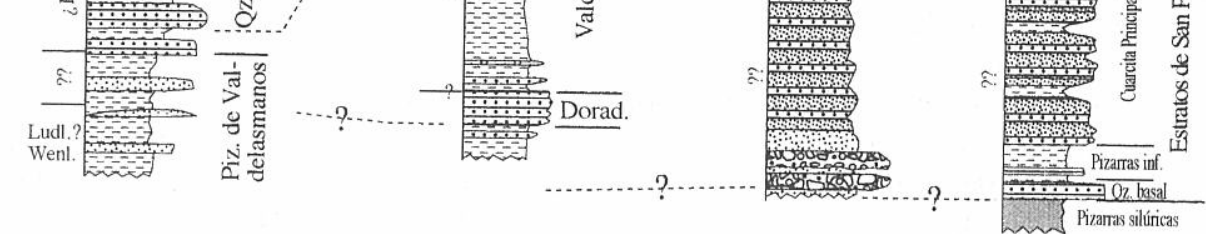

Sierra Morena Central-Montes de Toledo Sierra Morena Oriental

Figura 4. Correlación aproximada entre las series devónicas “tipo" de cuatro áreas de la Zona Centroibérica meridional, y del sinclinal de La Codosera-Portalegre (Z. Ossa-Morena). Las columnas, de izquierda a derecha, están ordenadas de ONO a ESE.

con los hiatos representados por costras ferruginosas en la Fm. Risquillo. Por su parte, la unidad cuarcítica superior, podría correlacionarse con el Mb. Puerto del Ciervo, de la Fm. Valmayor y quizás con otras formaciones frasnienses del área de Almadén (Fig. 4). Butenweg (1968) citó en dicha unidad formas de la Biozona Almadenensis.

Los términos más altos de la sucesión devónica en Sierra Morena oriental, afloran en el área de La Carolina (Fig. 1), donde las "Pizarras Aquisgrana" contienen ostrácodos del Fameniense Superior (Charpentier et al., 1976). Los mismos autores incluyen todavía en el Fameniense las "Cuarcitas Campana" suprayacentes y atribuyen el resto de la Fm. Campana al Turnesiense-Viseense (Fig. 4).

La ausencia de los "Estratos de San Pablo" por debajo de la Fm. Campana en el área de La Carolina, señalada por Charpentier et al. (1976) y Gutiérrez Marco et al. (1990) puede deberse a la intensa tectonización del flanco norte del sinclinal de Los Pedroches, donde son frecuentes grandes fracturas en dirección de capa afectando al contacto entre el Carbonífero de Los Pedroches y las restantes series paleozoicas.

La serie devónica de Sierra Morena Oriental tiene todavía niveles inéditos, como lo demuestra el hallazgo, actual- mente en estudio, de areniscas cuarcíticas ferruginosas en el área de Almedina, con braquiópodos (Eoparaphorhynchus cf. triaequalis) del Fameniense Inferior, sin equivalentes en el resto de la ZCI. Este hallazgo apunta a la existencia de condiciones más someras en esta región, durante el Fameniense, que las representadas en el área de Almadén por las pizarras con nódulos de Guadalmez.

En el extremo oeste de la ZCI meridional, el Devónico aflora en los sinclinales de Cáceres y Sierra de San Pedro (Figs. 1, 4). El más completo y mejor conocido es este último, con un espesor estimado de unos $1.500 \mathrm{~m}$. (Soldevila, 1992b). Las asociaciones faunísticas son totalmente comparables con las de otros afloramientos de la misma edad de la ZCI meridional. El Devónico Inferior (Emsiense) ha sido documentado en las "Cuarcitas de Aliseda", al menos en una localidad (Brachyspirifer, Euryspirifer, Arduspirifer, Uncinulus, Schizophoria, Plebejochonetes y otros). El Frasniense, en cambio, está representado por numerosos yacimientos. Las asociaciones fósiles identificadas tienen, sin embargo, una distribución anómala en la estratigrafía de Soldevila (1992b, 370-374, Fig. 4), que podría reflejar la intrincada tectónica de la región. En cualquier caso, dichas asociaciones 
fósiles acreditan, como mínimo, la presencia de las Biozonas Almadenensis y Muelleri. Hacia el km. 28,3 de la carretera de Cáceres a Badajoz, se encontró un interesante yacimiento en pizarras satinadas, gris claro a rosadas, con braquiópodos inarticulados y articulados, entre los últimos: Longispina n. sp. A, Apousiella sp., Ripidiorhynchus sp., homocténidos abundantes: Homoctenus sp., ostrácodos entomozoidos y de otros tipos, e icnofósiles, del Frasniense Medio o Superior. Esta asociación fósil aparece en una facies muy similar y tiene varios elementos comunes con la de las pizarras frasnienses de Alange, en la ZOM (ver luego). La mayoría de yacimientos asignables al Fameniense, pertenecen al Fameniense Superior, con braquiópodos abundantes (Cyrtospiriferidae, Hispidaria, Torynifer, Centrorhynchus, Composita, Cleiothyridina y otros) aunque, generalmente, mal conservados, en facies grauváquicas y microconglomeráticas, con pizarras intercaladas. La falta de cualquier indicación bioestratigráfica del Devónico Medio, permite sospechar que en este área se desarrolla también la extensa laguna mesodevónica conocida en el resto de la ZCI.

\section{OBSERVACIONES COMPLEMENTARIAS ACERCA DEL DEVÓNICO DEL "DOMINIO" OBEJO-VALSEQUILLO-PUEBLA DE LA REINA (ZONA OSSA-MORENA SEPTENTRIONAL)}

[Los datos que consignamos a continuación, salvo indicación expresa en diferente sentido, proceden de los trabajos realizados para el Proyecto MAGNA 1:50.000, con la participación de JLG-A (braquiópodos y bivalvos), M. A. Arbizu (trilobites), M. Truyóls-Massoni (tentaculitoideos), F. Soto (corales)].

Como se ha indicado anteriormente, el "dominio" Obejo-Valsequillo-Puebla de la Reina (Delgado et al., 1977), comprendería el conjunto de afloramientos devónicos dispersos que se extienden entre la zona de cizalla Badajoz-Córdoba y el Sinclinal de Los Pedroches y estructuras equivalentes de su prolongación occidental, incluyendo el sinclinal de La Codosera-Puebla de Obando (Soldevila 1992a) y el área portuguesa de Portalegre y Dornes (Perdigão, 1967, 1973, 1974, 1979) (Figs. 1, 4)

En esta región, el Devónico Inferior tiene una representación paleontológica mucho más completa que en la parte meridional de la ZCI, y comprende elementos desconocidos o raros en dicha Zona. El Lochkoviense se desarrolla en areniscas ferruginosas y areniscas amarillentas con lentejones de calizas bioclásticas, caracterizadas por la presencia de braquiópodos, entre ellos, pequeños rinconélidos asignables a Microsphaeridiorhynchus? sp., y Hebetoechia cf. nitidula, Platyorthis gr. monnieri, Protocortezorthis cf. trigeri, Howellella (Howellella) cf. mercuri, Mutationella cf. barroisi, Xana n. sp., Mclearnitesella sp., Protathyris gr. praecursor, bivalvos: Actinopteria sp., Leiopteria (Leiopteria) sp., Modiomorpha sp., Megambonia sp., Sphenotus ? sp., corales pleurodíctidos: Cleistopora geometrica, y trilobites muy característicos: Acastella jacquemonti, A. tanzidensis y Digonus sp. El Praguiense se presenta en facies de areniscas carbonatadas y pizarras, con frecuentes intercalaciones de cali- zas arenosas, bioclásticas, a veces coralinas (principalmente tabulados favosítidos: Thamnopora spp., Favosites spp., y otros). Los principales elementos fósiles son braquiópodos: Schizophoria spp., Platyorthis spp., Pleurochonetes oehlerti, Plebejochonetes sp., Fascistropheodonta sp., Straelenia sp., Hexarhytis spp., Brachyspirifer cf. becki, B. cf. williamsoni, Uncinulus gr. subwilsoni, Acrospirifer sp., Hysterolites cf. dolosus, Mauispirifer n. sp. H, Tenuicostella sp., Corylispirifer sp., Cyrtina spp., Paulinella gr. guerangeri, P. gaudryi, Meganteris spp., Mutationella sp. y tentaculitoideos: Nowakia acuaria, Tentaculites gr. straeleni. El Emsiense, desarrollado en facies similares a las anteriores, comprende principalmente braquiópodos: Schizophoria sp., Leptostrophiella explanata, Plicostropheodonta aff. diffusa, Bojodouvillina sp., Oligoptycherhynchus gr. subpareti, Uncinulus sp., Brachyspirifer gr. carinatus, Alatiformia? sp., Euryspirifer pellicoi, y otros. En Portalegre y Dornes, Perdigão (1967, 1979) describió, asimismo, importantes asociaciones fósiles del Emsiense Superior con, entre otros, Platyorthis circularis, Schizophoria vulvaria, Tyersella sp., Zdimir spp., Protodouvillina cf. taeniolata, Teichostrophia sp., Loreleiella sp., "Uncinulus pila", "U. orbignyanus", Arduspirifer sp., Euryspirifer sp., Paraspirifer gr. cultrijugatus y Rhenothyris gr. curvata.

El Frasniense se conoce en muy pocas localidades y comprende elementos conocidos en la ZCI (unidad de Valdeborracho, Soldevila, 1992a). Las asociaciones fósiles del Fameniense corresponden, en general, al Fameniense Superior. Los fósiles suelen aparecer en areniscas amarillentas con cemento calcáreo, donde abundan los braquiópodos: Praewaagenoconcha sp., Chonopectus? sp., "Rugosochonetes" aff. kennedyensis, Centrorhynchus aff. letiensis, Gastrodetoechia sp., Cyrtospirifer spp., y los bivalvos aviculopectínidos.

El Devónico Medio está ausente en parte del "dominio" Obejo-Valsequillo-Puebla de la Reina, denotando que en la Zona Ossa-Morena se desarrolla también una gran laguna estratigráfica intradevónica.

Una notable excepción frente a este modelo, es la sucesión devónica de los alrededores de Alange y su prolongación oriental hasta, por lo menos, el término de Valsequillo (Fig. 1). En efecto, en este área el Devónico reposa discordantemente sobre el Silúrico (Wenlockiense-Ludloviense; Gutiérrez Marco, com. pers.). La parte inferior (que en Alange se ha denominado Formación La Manchuela; Racheboeuf et al., 1986) está constituida por areniscas ferruginosas, con intercalaciones de pizarras oscuras con lentejones de calizas arenosas; lateralmente las calizas llegan a hacerse muy importantes, en especial cerca del extremo oriental del área, en la Sierra del Pedroso. Las asociaciones fósiles de estos niveles, están constituidas sobre todo por braquiópodos: Plicostropheodonta sp., Fascistropheodonta sp., Pleurochonetes cf. aulnensis, Ctenochonetes sp., Athyrididae, Brachyspirifer sp., Hysterolites sp., Tenuicostella sp., Paulinella cf. gerangeri, Globithyris ? oliviani, Meganteris sp., y trilobites: Pseudocryphaeus sp. y tentaculítidos, del Praguiense, y corales datados como del Emsiense Sup. por Rodríguez (1978) y Rodríguez y Soto (1979). La parte superior está constituida por pizarras sericíticas con finos niveles de areniscas blancas y rojizas hacia la base. En dicha parte de la sucesión, existe una interesante asociación fósil del Givetiense (posiblemente 
Superior) en varias localidades de las hojas geológicas de Almendralejo y Oliva de Mérida, quizás el más claro exponente paleontológico del Devónico Medio en toda la región mariánica, con braquiópodos: Iridistrophia ? sp., Eostrophalosia sp., Devonochonetes aff. kerfornei, Dagnachonetes sp., Longispina sp., Cupularostrum ? sp., Nucleospira sp., Ilmenia aff. subhians, Carpinaria sp., trilobites: Bradocryphaeus sp., tentaculítidos: Tentaculites sp., bivalvos, crinoideos, ostrácodos, briozoos e icnofósiles. Más al ESE, en la hoja de Valsequillo, se han citado también corales probablemente givetienses: Temnophyllum richardsoni, Tabulophyllum traverense, Diplochone sp. y Disphyllum pedrosensis (Rodríguez y Soto, 1979). Las asociaciones fósiles de las pizarras sericíticas comprenden también interesantes formas del Frasniense y, quizás, de la misma base del Fameniense (Racheboeuf et al., 1986), como braquiópodos inarticulados y articulados, entre los últimos: Monelasmina ? sp. y Longispina n. sp. A (non Pradochonetes muelleri, sensu Racheboeuf et al., 1986), cefalópodos: Bactrites sp., Manticoceras sp. y Aulatornoceras sp., bivalvos: Buchiola palmata, B. gr. kaisini, B. spp., Guerichia venusta, hiolítidos, homocténidos: Homoctenus aff. tenuicinctus tenuicinctus, $H$. cf. ultimus, ostrácodos: Entomozoe (Nehdentomis) cf. pseudorichterina, Entomoprimitia (Entomoprimitia) sp., Richterina sp., R. (Maternella) spp., trilobites: Heliopyge sp. y crustáceos filocáridos.

De acuerdo con los aspectos señalados, el Devónico de Alange se acercaría al de la ZCI, con semejanzas sorprendentes durante el Frasniense más alto con el de la Sierra de San Pedro (ver antes), aunque faltan las abundantes y ubicuas asociaciones fósiles de espiriféridos tan típicas en dicha Zona.

\section{AGRADECIMIENTOS}

Los Dres. Peter Carls y Juan Carlos Gutiérrez Marco, revisores del escrito original, realizaron valiosas sugerencias y correcciones al texto de esta publicación. A los dos queremos agradecer el que dedicaran parte de su tiempo y sus conocimientos en mejorar esta síntesis.

\section{BIBLIOGRAFÍA}

Aldaya, F., Carls, P., Martínez García, E. et Quiroga, J. L. 1976. Nouvelles précisions sur l'age de la série de San Vitero (Zamora, Nord Ouest de L'Espagne). Comptes rendus de l'Academie des Sciences de Paris, (D), 283, 881-883.

Almela, A., Alvarado, M., Coma, J., Felgueroso, C. y Quintero, I. 1962. Estudio geológico de la región de Almadén. Boletín del Instituto Geológico y Minero de España, 73, 197-327.

Bultynck, P. et Soers, E. 1971. Le Silurien superieur et le Devonien inferieur de la Sierra de Guadarrama (Espagne centrale). Bulletin de l'Institut royal des Sciences naturelles de Belgique, 47 (1), 1-22.

Butenweg, P. 1968. Geologische Untersuchungen im Ostteil der Sierra Morena nordöstlich von La Carolina (Provinz Jaén, Spanien). Münstersche Forschungen Geologie Paläontologie, 6, 1-125.

Calvo, A. A. 1993. Conodontos del Devónico Inferior en el sinclinal de Herrera del Duque (Badajoz, So de España). Coloquios de Paleontología, 45, 9-227.
Carls, P. 1988. The Devonian of Celtiberia (Spain) and Devonian paleogeography of SW Europe. In: Devonian of the world (Proceed. 2nd Int.Symp.Devonian System, Calgary, 1987) (Eds. N.J.McMillan, A.F.Embry y D.J.Glass), Canadian Society Petroleum Geology Memoirs, 14 (1), 421-466.

Charpentier, J. L., Lethiers, F. et Tamain, G. 1976. Les " Schistes Aquisgrana" à Ostracodes du Dévonien supérieur-terminal en Sierra Morena Orientale (Espagne). Annales de la Societé Géologique du Nord, 36, 353-362.

Delgado-Quesada, M., Liñán, E., Pascual, E. y Pérez-Lorente, F. 1977. Criterios para la diferenciación de Dominios en Sierra Morena Central. Studia Geologica, Universidad de Salamanca, 12, 75-90.

Díez-Balda, M. A., Vegas, R. and González-Lodeiro, F. 1990. Part IV. Central-Iberian Zone. 2.2. Structure. In: Pre-Mesozoic Geology of Iberia (Eds. R. D. Dallmeyer y E. Martínez-García). Springer-Verlag, Berlin, 172-188.

García-Alcalde, J. L., Arbizu M. A., Pardo Alonso, M. V. y García-López, S. 1984. El límite Devónico-Carbonífero en el área de Guadalmez-Santa Eufemia (Provs. de Ciudad Real y Córdoba Sierra Morena España). I Congreso Español de Geología. Segovia, 1, 421-430.

García-Alcalde, J. L., Arbizu, M., García-Lopez, S., Leyva, F., Montesinos, R., Soto, F. and Truyóls-Massoni, M. 1990. Devonian stage boundaries (Lochkovian/Pragian, Pragian/Emsian, and Eifelian/Givetian) in the Cantabric region (NW Spain). Neues Jahrbuch für Geologie und Paläontologie Abhandlungen, 180 (2), 177-207.

Groth, J. 1911. Sur le Primaire de la Sierra Morena. Comptes rendus de l'Academie des Sciences de Paris, 152, 231-232.

Groth, J. 1914. Les schistes à Goniatites de Guadalmez. Comptes rendus de l'Academie des Sciences de Paris, 158, 525-526.

Gutiérrez Marco, J.C., San José, M. A. de and Pieren, A. P. 1990. 2.1.3. Post-Cambrian Palaeozoic Stratigraphy. In: Pre-Mesozoic Geology of Iberia (Eds. R. D. Dallmeyer y E. Martínez-García). Springer-Verlag, Berlin, 160-171.

Herranz Araújo, P. 1994. Las discontinuidades estratigráficas principales en el sector central del NE de «Ossa-Morena»: Rango y significado tectosedimentario. Memórias e Notícias. Publicaçoes do Museu e Laboratório Mineralógico e Geológico da Universidade de Coimbra, 97, 51-80.

Jaeger, H. et Robardet, M. 1979. Le Silurien et le Dévonien basal dans le Nord de la province de Sevilla (Espagne). Géobios, 12, 687-714.

Julivert, M., Fontboté, M., Ribeiro, A. y Conde, L. E. 1974. Memoria explicativa del Mapa Tectónico de la Península Ibérica y Baleares. E. 1:1.000.000. Instituto Geológico y Minero de España, Madrid, 1-113.

Julivert, M., Truyóls, J. y Vergés, J. 1983. El Devónico del Macizo Ibérico. En: Geología de España, Libro Jubilar J. M. Ríos. Instituto Geológico y Minero de España, 1, 265-311.

Kettel, D. 1968. Zur Geologie der östlichen Sierra Morena im Grenzbereich der Provinzen Jaén, Ciudad Real und Albacete (Spanien). Münstersche Forschungen Geologie Paläontologie, 8, 1-159.

Lotze, F. 1945. Zur Gliederung der Varisziden de Iberischen Meseta. Geotektonische Forschungen, 6, 78-92.

Maass, R. 1961. Die Geologie, insbesondere das Devon im Bereich der Orte Castuera-Cabeza del Buey-Monterrubio (Extremadura, Südspanien). Akademie der Wissenschaften und der Litera- 
tur. Abhandlungen der Mathematisch-Naturwissenschaftelichen Klase, 1961 (2), 60-84.

Martínez Catalán, J. R. 1990. West Asturian-Leonese Zone. Introduction. In: Pre-Mesozoic Geology of Iberia (Eds. R.D. Dallmeyer y E. Martínez-García). Springer-Verlag, Berlin, 91

Müller, W. 1929. Die Fauna der Frasnes-Stufe bei Almadén (Sierra Morena, Spanien). Abh. Senck. Naturf. Ges., 41 (5), 243-282.

Oczlon, M. S. 1990. Ocean currents and unconformities: the North Gondwana Middle Devonian. Geology, 18 (6), 509-512.

Oliveira, J. R., García-Alcalde, J. L., Liñán, E. and Truyóls, J. 1986. The Famennian of the Iberian Peninsula. Annales de la Société Géologique de Belgique, 109, 159-174.

Pardo Alonso, M. V. and De Renzi, M. 1993. Presence of stromatolites in the Lower Devonian of Herrera del Duque (Central-Iberian Zone, Spain). Comunicações da XII Reunião de Geologia do Oeste Peninsular, 1, 29-40.

Pardo Alonso, M. V. y García-Alcalde J. L. 1984a. El Devónico de la región de Almadén (Ciudad Real, España). I Congreso Español de Geología, Segovia, 1, 473-482.

Pardo Alonso, M. V. y García-Alcalde J. L. 1984b. Biostratigrafía del Devónico de la región de Almadén (Ciudad Real, España). Trabajos de Geología, Universidad de Oviedo, 14, 79-120.

Perdigão, J. C. 1967. Descoberta de Mesodevónico em Portugal (Portalegre). Comunicações dos Serviços Geológicos de Portugal, 52, 27-48.

Perdigão, J. C. 1973. A fauna dos grés e quartzitos silúrico-devónicos de Portalegre e a sua posição estratigráfica. Comunicações dos Serviços Geológicos de Portugal, 56, 5-32.

Perdigão, J. C. 1974. O Devónico de Portalegre. Comunicações dos Serviços Geológicos de Portugal, 57, 203-228.

Perdigão, J. C. 1979. O Devónico de Dornes (Paleontologia e estratigrafia). Comunicações dos Serviços Geológicos de Portugal, 65, 193-199.

Prado, C. de, Verneuil, E. de et Barrande, J. 1855. Mémoire sur la géologie d'Almaden, d'une partie de la Sierra Morena et des montagnes de Tolède, suivi d'une description des fossiles qui s'y rencontrent. Extrait du Bulletin de la Société Géologique de France, 2 ser., 12 , 1-86.

Puschmann, H. 1967. Zum Problem der Schichtlücken im Devon der Sierra Morena (Spanien). Geologische Rundschhau, 56, 528-542.

Puschmann, H. 1970. Das Paläozoikum der Nördlichen "Sierra Morena" am Beispiel der Mulde von Herrera del Duque (Spanien). Geologie, 19 (3), 309-329.

Racheboeuf, P. R., Lethiers, F., Babin, C., Rolfe, W. D. I. et Marez, E. de 1986. Les faunes du Dévonien supérieur d'Alange (pro- vince de Badajoz, Sud-Ouest de l'Espagne). Géologie Méditerranéenne, 12-13 (1-2), 37-47.

Racheboeuf, P. R. et Robardet, M. 1986. Le Pridoli et le Dévonien inférieur de la Zone d'Ossa-Morena (sud-ouest de la Peninsule Iberique. (Etude des brachiopodes). Geologica Palaeontologica, 20, 11-37.

Robardet, M., Blaise, J., Bouyx, E., Gourvennec, R., Lardeux, H., Le Herisse, A., Le Menn, J., Melou, M., Paris, F., Plusquellec, Y., Poncet, J., Regnault, S., Rioult, M. et Weyant, M. 1993. Paléogéographie de l'Europe occidentale de l'Ordovicien au Dévonien. Bulletin de la Société Géologique de France, 164 (5), 683-695.

Robardet, M. 1976. Originalité du segment hercynien sud-ibérique au Paléozöique inférieur: Ordovicien, Silurien et Devonien dans le Nord de la Province de Séville (Espagne). Comptes rendus de l'Academie des Sciences de Paris, 283, 999-1002.

Rodríguez-García, S. 1978. Corales Rugosos del Devónico de la Sierra del Pedroso. Estudios Geológicos, 34, 331-350.

Rodríguez-García, S. y Soto, F. 1979. Nuevos datos sobre los corales rugosos del Devónico de la Sierra del Pedroso. Estudios Geológicos, 35, 345-354.

Rodríguez-Núñez, V. M., Gutiérrez Marco, J. C. y Sarmiento, G. N. 1989. Rasgos bioestratigráficos de la sucesión silúrica del Sinclinal de Guadarranque (provincias de Cáceres, Badajoz y Ciudad Real). Coloquios de Paleontología, 42, 83-106.

Soldevila, J. 1992a. La sucesión paleozoica en el sinforme de la Codosera-Puebla de Obando (Provincias de Cáceres y Badajoz, SO de España). Estudios Geológicos, 48, 353-362.

Soldevila, J. 1992b. La sucesión paleozoica en el sinforme de la Sierra de San Pedro (Provincias de Cáceres y Badajoz, SO de España). Estudios Geológicos, 48, 363-379.

Truyóls-Massoni, M. y Quiroga, J. L. 1982. Tentaculites Dacrioconáridos en el sinforme de Alcañices (Prov. de Zamora). Cuadernos do Laboratorio Xeolóxico de Laxe, 2, 171-175.

Walter, R. (con contribuciones de W. Assmann, H. G. Bochmann, R, Hollinger, H. Kalthoff, H. J. Kelch, L. Laus, R. Maass, E. Machens, R. Merten, M. Ransweiler, K. Redlin, H. J. Schmidt, O. Suhr y K. Oeggen). 1977. Zwei geologische Traversen durch die südliche Iberische Meseta, Spanien. Münstersche Forschungen Geologie Paläontologie, 42: 1-55.

Weyant, M., Brice, D., Racheboeuf, P. R., Babin, C. et Robardet, M. 1988. Le Dévonien supérieur du synclinal du Valle (province de Seville, Espagne). Revue de Paléobiologie, 7 (1), 233-260.

Manuscrito recibido: 1 de diciembre, 1994 Manuscrito aceptado: 1 de septiembre de 1995 